\title{
Mood disorders and migration
}

\author{
Meta-analysis
}

SANNE G. H. A. SWINNEN and JEAN-PAUL SELTEN

Background Migration is a risk factor
for the development of schizophrenia.

\begin{abstract}
Aims To examine whether migration is also a risk factor for bipolar affective disorder, unipolar depressive disorder and mood disorders in general.
\end{abstract}

\begin{abstract}
Method Medline was searched for population-based incidence studies concerning mood disorders among migrants and mean relative risks were computed using a mixed-effects statistical model.
\end{abstract}

Results Only a few studies of unipolar depressive disorder were retrieved. The mean relative risk of developing bipolar affective disorder among migrants was 2.47 (95\% Cl I.33-4.59). However, after excluding people of African-Caribbean origin in the UK this risk was no longer significantly increased. The mean relative risk of mood disorders of unspecified polarity was I.25 (95\% Cl I.04-1.49) and that of any mood disorder was 1.38 (95\% Cl1.17-1.62).

\section{Conclusions There is no conclusive} evidence for a large increase in the risk of mood disorders associated with migration.

\section{Declaration of interest None.}

Meta-analysis has found a personal or family history of migration to be an important risk factor for the development of schizophrenia, with a mean weighted relative risk of 2.9 (95\% CI 2.5-3.4) (CantorGraae \& Selten, 2005). There are also reports of an increased incidence of bipolar affective disorder among migrants (Leff $e t$ al, 1976; van Os et al, 1996; Lloyd et al, 2005). The aim of the present meta-analysis was to determine, on the basis of population-based incidence studies of mood disorders among migrant groups, the magnitude of the risk of bipolar affective disorder, unipolar depressive disorder, and mood disorders of unspecified polarity associated with migration.

\section{METHOD}

\section{Selection of studies}

A systematic Medline search (1966 to June 2005) was conducted using the keywords migration, ethnicity, bipolar disorder, depressive disorder, mood disorders and various synonyms for these search terms. Bibliographies from selected publications were cross-referenced. To be included in the meta-analysis a study had to meet the following criteria:

(a) it had to be a population-based incidence study, i.e. it reported incidence rates for one or more migrant groups residing in a circumscribed area, or provided numerators and denominators for such calculations to be made;

(b) the study corrected the results for age differences between the migrant and reference groups, or provided data that made this correction possible;

(c) the study was published in an English-language, peer-reviewed scientific journal.

In this meta-analytic review we used the term 'migrant' for people who were born abroad or whose parents were foreign-born (first- and second-generation migrants respectively) and for people from minority ethnic populations defined on the basis of skin colour, who can be assumed to be firstor second-generation migrants (e.g. Black Africans in the UK). Denominators for studies conducted in the UK were estimated from national census data, with two studies using the 'remainder of the general population' (Harrison et al, 1988; van Os et al, 1996) and two other studies using the category 'White' (McGovern \& Cope, 1987; Lloyd et al, 2005) as reference groups; this implies that some members of the reference group might actually be immigrants to the UK, whereas some Black people or members of other minority ethnic populations might have lived in the country for more than two generations. However, as this type of classification error would probably lead to a decrease in the computed effect size, we accepted these divergent categorisations.

We excluded some identified studies because they were not population-based (e.g. Pope et al, 1983; Grove et al, 1986), and other studies because age-specific rates were not reported and correction for age differences between groups could not be performed (e.g. King et al, 1994). There were too few studies and effect sizes to justify a separate meta-analysis on unipolar depressive disorder. Thus, we computed the mean weighted relative risk of bipolar affective disorder (meta-analysis 1), mood disorders of unspecified polarity (metaanalysis 2) or any mood disorder (metaanalysis 3 ) associated with migration. This final analysis incorporated the studies on bipolar affective disorder, unipolar depressive disorder and mood disorders of unspecified polarity. Possible gender effects were examined in analyses $1 \mathrm{~A}, 2 \mathrm{~A}$ and $3 \mathrm{~A}$. The effect sizes selected for meta-analysis 1 (bipolar affective disorder) concerned the ICD-9 (World Health Organization, 1978) diagnoses manic-depressive psychosis, manic type (ICD-9 code 296.0) or circular type (296.2, 296.3, 296.4, 296.5), or the ICD-10 (World Health Organization, 1992) diagnosis of bipolar affective disorder (ICD-10 code F31).

For meta-analysis 2 we used the results of studies applying the ICD-9 diagnostic category of manic-depressive psychosis without further specification (code 296) (Thomas et al, 1993; Mortensen et al, 1997; Sundquist et al, 2004), or using the non-standardised diagnosis 'affective disorder' (e.g. Hemsi, 1967). One study distinguished between affective illness and 
'reactive depression' (Rwegellera, 1977) and in order to be consistent we combined the results of these two diagnostic groups to one effect size, to be included in meta-analysis 2 . The studies concerning unipolar depressive disorder applied the term reactive depression (Rwegellera, 1977) or the ICD-9 diagnosis of manicdepressive psychosis, depressed type (296.1) (Selten et al, 2003). In order to avoid the weighing of Rwegellera's 'reactive depression' twice, this separate category was not added to meta-analysis 3 , but incorporated only once as the abovementioned combined figure.

Unfortunately there were insufficient effect sizes to consider first- and secondgeneration migrants as separate groups. Consequently, the meta-analyses incorporated studies that did not discriminate between both generations and studies that presented figures for the first generation only.

\section{Meta-analysis}

The two authors performed the extraction of data and calculation of relative risks separately, with consensus being reached when there was initial disagreement. From each study relative risks for one or more migrant groups were derived. Age- and gender- adjusted relative risks were calculated by Poisson regression analysis, using the available data on numerators and denominators.

Because the various studies presented information on numerators, denominators and rates differently, in order to use the same method of variance estimation we used the formula $V=1 / N_{n}+1 / N_{m}$, where $N_{\mathrm{n}}$ is the number of native-born patients and $N_{\mathrm{m}}$ the number of foreign-born patients; this was derived from the formula for the variance of odds ratios (Lipsey $\&$ Wilson, 2001), as previously done in meta-analyses (e.g. Cantor-Graae \& Selten, 2005). To prevent studies with large population samples from dominating the analyses, we set the number of patients in studies with more than 500 participants at 500, as suggested by Shadish \& Haddock (1994). In order to examine whether the various effect sizes that were averaged into a mean value all estimated the same population effect size, a homogeneity test based on the $Q_{\mathrm{w}}$ statistic was performed. Significant values for $Q_{\mathrm{w}}$ indicate a heterogeneous distribution across studies, i.e. the variability of the effect sizes was larger than would be expected from sampling error alone. We used the $Q_{\mathrm{B}}$ statistic to test whether differences in effect sizes between male and female migrants were statistically significant (Lipsey \& Wilson, 2001). Since the distribution of the effect sizes remained heterogeneous even after modelling betweenstudy differences, we performed additional analyses using a mixed-effects model. A mixed-effects model assumes that variability in the effect size distribution is due to systematic between-study differences, subjectlevel sampling error and an additional random component (Lipsey \& Wilson, 2001). All analyses were carried out using the MetaWin 2.0 statistical package (Rosenberg et al, 2000).

\section{RESULTS}

Fourteen studies met our inclusion criteria; the majority had a retrospective case-note design and concerned first psychiatric hospital admission. Surprisingly, only two studies concerned depressive disorders (Rwegellera, 1977; Selten et al, 2003). Five studies (11 effect sizes) that investigated the relationship between bipolar affective disorder and migration were included in the first meta-analysis (Leff et al, 1976; Harrison et al, 1988; van Os et al, 1996; Selten et al, 2003; Lloyd et al, 2005). Four of these studies had taken place in the UK and one in The Netherlands (Selten et al,

Table I Meta-analyses of population-based incidence studies investigating the relationship between migration and the development of mood disorders

\begin{tabular}{|c|c|c|c|c|c|c|}
\hline & $\begin{array}{l}\text { Number of } \\
\text { effect sizes }\end{array}$ & $\begin{array}{c}\text { Relative } \\
\text { risk }\end{array}$ & $(95 \% \mathrm{Cl})$ & $Q_{w}^{\prime}$ & $Q_{\mathrm{B}}^{2}$ & $P^{3}$ \\
\hline \multicolumn{7}{|l|}{ Analysis I } \\
\hline Analysis I: bipolar affective disorder & II & 2.47 & $(1.33-4.59)$ & 10.54 & & 0.39 \\
\hline Analysis I excluding African-Caribbeans & 8 & 1.75 & $(0.94-3.28)$ & 8.91 & & 0.26 \\
\hline Analysis IA: gender & & & & & 1.22 & 0.27 \\
\hline Male & 9 & 2.51 & $(1.34-4.72)$ & & & \\
\hline Female & 9 & 1.62 & $(0.85-3.12)$ & & & \\
\hline \multicolumn{7}{|l|}{ Analysis 2} \\
\hline Analysis 2: mood disorders of unspecified polarity & 17 & 1.25 & $(1.04-1.49)$ & 27.77 & & 0.03 \\
\hline Analysis 2 excluding African-Caribbeans & 12 & 1.20 & $(\mathrm{I} .0 \mathrm{I}-\mathrm{I} .42)$ & 19.06 & & 0.06 \\
\hline Analysis 2A: gender & & & & & 0.28 & 0.60 \\
\hline Male & II & 0.97 & $(0.69-1.35)$ & & & \\
\hline Female & II & 1.08 & $(0.77-I .5 I)$ & & & \\
\hline \multicolumn{7}{|l|}{ Analysis 3} \\
\hline Analysis 3: any mood disorder & 34 & 1.38 & $(1.17-1.62)$ & 68.94 & & 0.0002 \\
\hline Analysis 3 excluding African-Caribbeans & 25 & 1.18 & $(1.0 \mathrm{I}-\mathrm{I} .38)$ & 48.53 & & 0.002 \\
\hline Analysis $3 \mathrm{~A}$ : gender & & & & & 1.23 & 0.27 \\
\hline Male & 24 & 1.36 & $(1.06-1.75)$ & & & \\
\hline Female & 24 & 1.13 & $(0.88-1.45)$ & & & \\
\hline
\end{tabular}

I. Within-category homogeneity statistic, d.f. $=$ number of effect sizes $-I$.

2. Between-category homogeneity statistic, d.f. $=I$.

3. Value for $Q_{W}$ or $Q_{B}$. 
2003). The second meta-analysis, on mood disorders of unspecified polarity, was based on nine studies (17 effect sizes) (Hemsi, 1967; Gershon \& Liebowitz, 1975; Rwegellera, 1977; Hitch \& Clegg, 1980; Krupinski \& Cochrane, 1980; McGovern \& Cope, 1987; Thomas et al, 1993; Mortensen et al, 1997; Sundquist et al, 2004), five of which were from the UK; the other studies had been conducted in Israel, Australia, Denmark and Sweden respectively. The characteristics of the included studies, plus the relative risks for the various migrant groups, are given as a data supplement to the online version of this paper. The results of the three meta-analyses and various sub-analyses are shown in Table 1.

The mean weighted relative risk of developing bipolar disorder among migrants compared with native-born people (metaanalysis 1) was 2.47 (95\% CI 1.33-4.59). Notwithstanding the substantial differences in effect sizes between various migrant groups, the homogeneity test based on the
$Q_{\mathrm{w}}$ statistic was not significant, which means that there was no evidence of a heterogeneous distribution across the included studies. However, with 11 effect sizes the power to show heterogeneity was limited. The second meta-analysis, covering the diagnostic group of mood disorders of unspecified polarity, yielded a mean weighted relative risk of $1.25(95 \%$ CI 1.04-1.49). However, there was significant heterogeneity across the studies. In the third meta-analysis, incorporating studies on bipolar affective disorder, unipolar depressive disorder and mood disorders of unspecified polarity (34 effect sizes), the mean relative risk of any mood disorder was 1.38 (95\% CI 1.17-1.62) and the heterogeneity was again significant.

The effect sizes and associated confidence intervals for the various migrant groups included in meta-analysis 3 are displayed in Fig. 1. Since many effect sizes concerned African-Caribbean people living in the UK, a population considered to be at a high risk of severe mental disorders, we repeated all analyses after exclusion of this group. The relative risk of bipolar affective disorder was then 1.75 (95\% CI 0.943.28 ), which was no longer significantly increased. In contrast, the increased risks of other mood disorders did not depend on the contribution of the African-Caribbean population (see Table 1). The mean relative risks of bipolar affective disorder, mood disorders of unspecified polarity and any mood disorder were not significantly greater for male migrants than for female migrants (analyses $1 \mathrm{~A}, 2 \mathrm{~A}$ and $3 \mathrm{~A}$ respectively: see Table 1 ).

We considered the possibility that our results were influenced by publication bias. Using the fail-safe calculation suggested by Rosenthal (1979), we calculated the number of non-significant and unpublished studies that would need to be added to metaanalysis 1,2 or 3 in order to change the results from significant to non-significant. These numbers were 47, 20 and 229

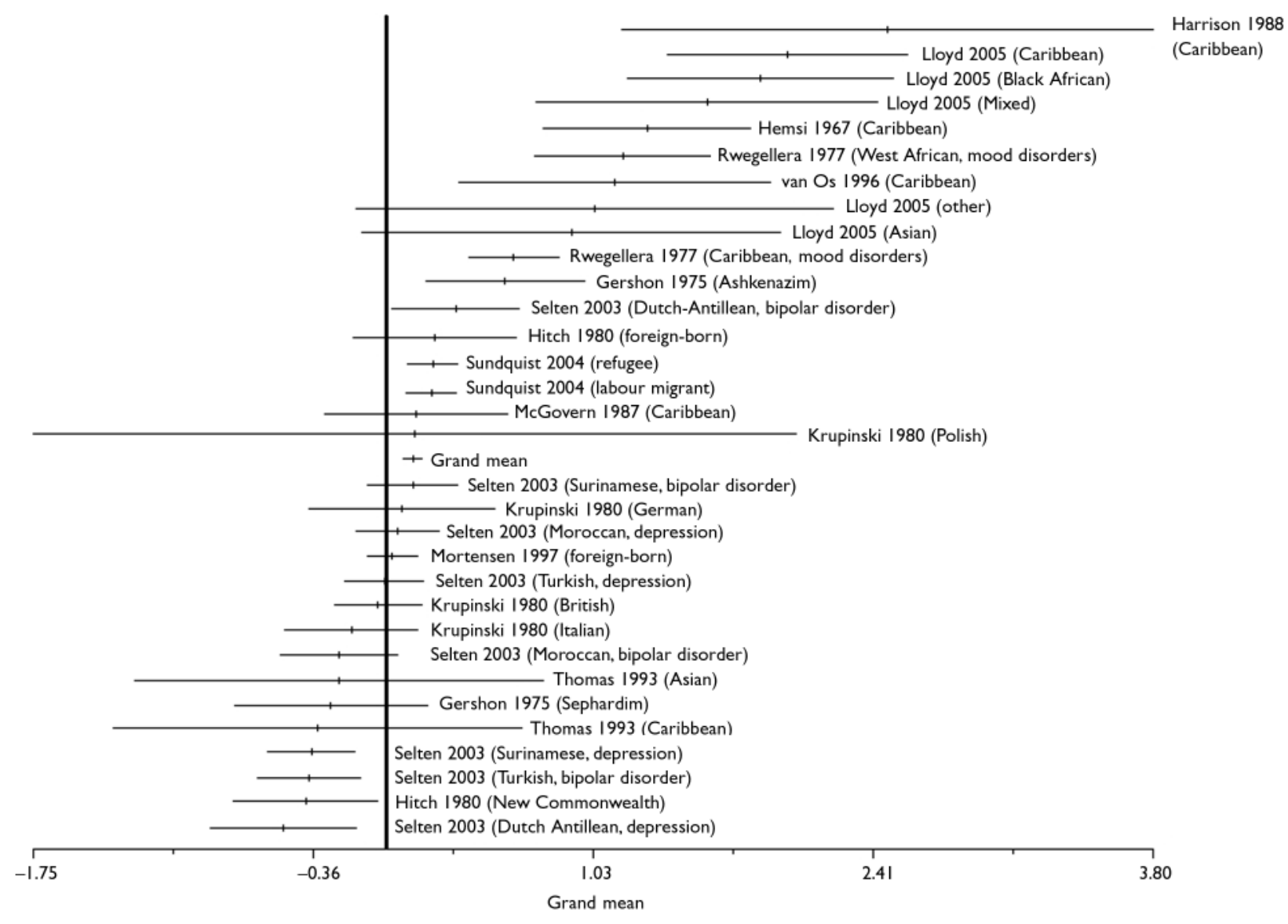

Fig. I Relative risks (and 95\% confidence intervals) of migrant groups included in population-based incidence studies (identified by first-named author and year) of the risk of mood disorders associated with migration. The figure shows the natural logarithms of all included effect sizes and the natural logarithm of the grand mean. The effect sizes concern studies of bipolar affective disorder, unipolar depressive disorder and mood disorders of unspecified polarity. If a study reported more than one effect size for a single immigrant group because different mood disorders had been examined, the type of mood disorder is specified. 
respectively. Thus, the results of metaanalyses 1 and 2 are unlikely to be explained by this kind of bias and the result of meta-analysis 3 cannot be attributed to it.

\section{DISCUSSION}

There is no conclusive evidence for a large increase in the risk of mood disorders among migrants. The mean weighted relative risk for the development of bipolar affective disorder associated with migration was found to be 2.47 (95\% CI 1.33-4.59). However, this result depended on the contribution of a single immigrant group - people of African-Caribbean origin living in the UK. The mean relative risk of developing any mood disorder among migrants was 1.38 (95\% CI 1.17-1.62). This effect did not depend on the contribution of African-Caribbean immigrants and could not be explained by publication bias. It is likely, therefore, that immigrants' risk of mood disorders is not increased as much as their risk of schizophrenia. Although it should be noted that there was significant heterogeneity in effect sizes, the mean weighted relative risk for schizophrenia associated with migration has been shown to be 2.9 (95\% CI 2.5-3.4) (Cantor-Graae \& Selten, 2005).

\section{Limitations}

\section{Numerators and denominators}

The number of population-based incidence studies concerning mood disorders among migrants was small and the number of countries in which they had been conducted was limited. Most of the included studies, particularly for the bipolar meta-analysis, reported incidence rates computed from small numerators. It can be argued that these rates should be treated with caution because of the limited numbers of migrant patients. Additionally, the denominators of studies conducted in the UK were based on national census data and there is concern about the quality of these data, in particular the possible underestimation of the number of young African-Caribbean men.

\section{Bias}

It has been suggested that reports of an elevated rate of bipolar disorder among migrants could be due to artefacts, such as overrepresentation of migrants in the agegroup with the highest incidence and inaccurate diagnosis (Bebbington \& Ramana,
1995). We eliminated the first possible source of bias by excluding studies that had not corrected for age differences between groups. However, diagnostic bias might have influenced our results: only two studies applied semi-structured diagnostic interviews (Harrison et al, 1988; Lloyd et al, 2005), and some studies did not use operationalised diagnostic criteria. Since several studies used data on first hospital admission, differences between the various ethnic groups in treatment-seeking and differences in admission practices might have biased the results. Furthermore, as shown by Kirov \& Murray (1999) and Kennedy et al (2004), African-Caribbean or African patients present more frequently with initial manic episodes, whereas White people with the same disorder more often present with depressive episodes. Since a manic episode leads more often to hospital admission than a depressive episode, this might lead to an overestimation of the risk of bipolar affective disorder among the African groups. Alternatively, it is possible that migrants with mood disorders are less likely to seek treatment compared with native-born people. It is conceivable, for example, that people from developing countries are less inclined to consider mood disorders as conditions that require medical treatment. The results of a prevalence study conducted in the UK, however, showed similar rates of seeking medical help for common mental disorders among AfricanCaribbean and White European populations. The same study showed that the 1-month prevalence of depressive disorders was only moderately increased among people of African-Caribbean origin (13\% v. 9\%; Shaw et al, 1999).

\section{Differences between various immigrant groups}

Our results indicate that the AfricanCaribbean population living in the UK is at particularly high risk of developing bipolar affective disorder but not unipolar depressive disorder. Remarkably, the exclusion of African-Caribbean individuals from meta-analyses 2 and 3 did not influence the results. It is noteworthy that the increased risk among these UK immigrant groups is not seen among Surinamese and Dutch Antillean migrants to The Netherlands (Selten et al, 2003). It is possible that the Dutch findings, which were based on hospital diagnoses and not on semi-structured diagnostic interviews, were biased. An alternative explanation is that African-Caribbean migrants living in the UK experience more difficulties, for example discrimination, than migrants from the same region living in The Netherlands. Indeed, the relative risks of schizophrenia for African-Caribbean and Black African people living in the UK are more increased than those for the Surinamese and Dutch Antillean populations in The Netherlands (Harrison et al, 1988; King et al, 1994; Selten et al, 2001).

\section{Interpretation}

Given the wealth of evidence that major life events precipitate the onset of mood disorders (e.g. Post, 1992), one would expect that the stress associated with the process of migration and adjustment to an unfamiliar, sometimes discriminatory and hostile, culture constitutes an important risk factor for mood disorders. At the same time, risk factors for depression such as poverty and low socio-economic status can be assumed to be more prevalent among many migrant populations. Consequently, it is difficult to understand why we found only a relatively small increased risk of mood disorders, compared with the increased risk of schizophrenia, among migrants. The difference could be explained by the selection hypothesis formulated by Ödegaard (1932), according to which people with a genetic predisposition for mood disorders develop strong attachments to people in their home countries and are less likely to migrate than people with a predisposition for schizophrenia. However, there is quite strong evidence against selection as the explanation for the association between schizophrenia and migration (Cantor-Graae \& Selten, 2005) and there is no evidence that selection accounts for the relatively small increase in the risk of mood disorders among immigrants.

The only mild increase in risk of mood disorders associated with migration, compared with the elevated risk of schizophrenia, may in part be due to the above-described differences in treatment-seeking and admission practices between the various ethnic groups. These factors are probably more variable for mood disorders than for schizophrenic disorders, which because of their severity will generally lead to hospitalisation. Still, finding other explanations for the association between migration and schizophrenia being stronger than that between migration and 
mood disorders remains an important challenge for future research.

\section{REFERENCES}

Bebbington, P. \& Ramana, R. (1995) The epidemiology of bipolar affective disorder. Social Psychiatry and Psychiatric Epidemiology, 30, 279-292.

Cantor-Graae, E. \& Selten, J. P. (2005) Schizophrenia and migration: a meta-analysis and review. American Journal of Psychiatry, 162, 12-24.

Gershon, E. S. \& Liebowitz, J. H. (1975) Sociocultura and demographic correlates of affective disorders in Jerusalem. Journal of Psychiatric Research, 12, 37-50.

Grove, W., Clayton, P. J., Endicott, J., et al (1986) Immigration and major affective disorder. Acta Psychiatrica et Neurologica Scandinavica, 74, 548-552.

Harrison, G., Owens, D., Holton, A., et al (1988) A prospective study of severe mental disorder in AfroCaribbean patients. Psychological Medicine, 18, 643-657.

Hemsi, L. K. (1967) Psychiatric morbidity of West Indian immigrants: a study of first admissions in London. Social Psychiatry, 2, 95-100.

Hitch, P. J. \& Clegg, P. (1980) Modes of referral of overseas immigrant and native-born first admissions to psychiatric hospital. Social Science and Medicine, I4A, 369-374.

Kennedy, N., Boydell, J., van Os, J., et al (2004) Ethnic differences in first clinical presentation of bipolar disorder: results from an epidemiological study. Journal of Affective Disorders, 83, 161-168.

King, M., Coker, E., Leavey, G., et al (1994) Incidence of psychotic illness in London. Comparison of ethnic groups. BMJ, 309, III9-1123.

Kirov, G. \& Murray, R. M. (1999) Ethnic differences in the presentation of bipolar affective disorder. European Psychiatry, 14, 199-204.

Krupinski, J. \& Cochrane, R. (1980) Migration and mental health - a comparative study. Journal of Intercultural Studies, I, 49-57.

Leff, J. P., Fischer, M. \& Bertelsen, A. (1976) A crossnational epidemiological study of mania. British journal of Psychiatry, 129, 428-442.

Lipsey, M. W. \& Wilson, D. B. (200I) Practical Metaanalysis. Sage.

SANNE G. H. A. SWINNEN, MD and JEAN-PAUL SELTEN, MD, PhD, Rudolf Magnus Institute of Neuroscience, Department of Psychiatry, University Medical Centre Utrecht, The Netherlands

Correspondence: Dr. J. P. Selten, University Hospital, PO Box 85500, 3508 GA Utrecht, Reference Number A00.24I, The Netherlands. Tel: + 3130 2508I80; fax: + 31302505443 ; email: j.p.selten@umcutrecht.nl

(First received 17 December 2005, final revision I5 August 2006, accepted I September 2006)

Lloyd, T., Kennedy, N., Fearon, P., et al (2005) Incidence of bipolar affective disorder in three UK cities: results from the $Æ$ $\mathrm{SOP}$ study. British Journal of Psychiatry 186, $|26-| 3 \mid$.

McGovern, D. \& Cope, R. (1987) First psychiatric admission rates of first and second generation Afro Caribbeans. Social Psychiatry, 22, 139-149.

Mortensen, P. B., Cantor-Graae, E., \& McNeil, T. F. (1997) Increased rates of schizophrenia among immigrants: some methodological concerns raised by Danish findings. Psychological Medicine, 27, 813-820.

Ödegaard, Ö. (1932) Emigration and insanity. Acta Psychiatrica et Neurologica Scandinavica Supplementum, 4, I-206.

Pope, H. G., lonescu-Pioggia, M. \& Yurgelun-Todd, D. (1983) Migration and manicdepressive illness. Comprehensive Psychiatry, 24, I58-165.

Post, R. M. (1992) Transduction of psychosocial stress into the neurobiology of recurrent affective disorder. American Journal of Psychiatry, 149, 999-1010.

Rosenberg, M. S., Adams, D. C. \& Gurevitch, J. (2000) MetaWin: Statistical Software for Meta-analysis (version 2.0). Sinauer.

Rosenthal, R. (1979) The 'file drawer problem' and tolerance for null results. Psychological Bulletin, 86, 638-641.

Rwegellera, G. G. C. (1977) Psychiatric morbidity among West Africans and West Indians living in London. Psychological Medicine, 7, 317-329.

Selten, J.-P., Veen, N., Feller, W., et al (200I) Incidence of psychotic disorders in immigrant groups to The Netherlands. British Journal of Psychiatry, 178, 367-372.

Selten, J.-P., van Os, J. \& Nolen, W. A. (2003) First admissions for mood disorders in immigrants to the
Netherlands. Social Psychiatry and Psychiatric Epidemiology, 38, 547-550.

Shadish, W. R. \& Haddock, C. K. (1994) Combining estimates of effect size. In The Handbook of Research Synthesis (eds H. Cooper \& L.V. Hedges), pp. 26I-280. Russell Sage.

Shaw, C. M., Creed, F., Tomenson, B., et al (1999) Prevalence of anxiety and depressive illness and help seeking behaviour in African Caribbeans and white Europeans: two phase general population survey. BMJ, 318, 302-305.

Sundquist, K., Frank, G. \& Sundquist, J. (2004) Urbanisation and incidence of psychosis and depression: follow-up study of 4.4 million women and men in Sweden. British Journal of Psychiatry, 184, 293-298.

Thomas, C. S., Stone, K., Osborn, M., et al (1993) Psychiatric morbidity and compulsory admission among UK-born Europeans, Afro-Caribbeans and Asians in central Manchester. British Journal of Psychiatry, I63, 91-99.

van Os, J., Takei, N., Castle, D. J., et al (1996) The incidence of mania: time trends in relation to gender and ethnicity. Social Psychiatry and Psychiatric Epidemiology, 31, $129-136$

World Health Organization (1978) International Statistical Classification of Diseases and Related Health Problems (ICD-9).WHO.

World Health Organization (1992) International Statistical Classification of Diseases and Related Health Problems (ICD-10).WHO. 